\author{
S. Ksouri ${ }^{\text {a,b }}$, M. Matmat ${ }^{\text {a }}$, H. Boukabache ${ }^{\text {a,b }}$, C.Escriba ${ }^{\text {a,b }}$, J.Y. Fourniols ${ }^{\text {a,b }}$. \\ ${ }^{\text {a }}$ CNRS ; LAAS ; 7 avenue du colonel Roche, F-31077 Toulouse, France \\ ${ }^{\mathrm{b}}$ Université de Toulouse ; UPS, INSA, INP, ISAE ; LAAS ; F-31077 Toulouse, France
}

\title{
DAMAGE DETECTION IN COMPOSITE LAMINATES AERONAUTICS STRUCTURES THROUGH ACCELEROMETERS NETWORK
}

\begin{abstract}
Over the past twenty years, the increase of use of structural materials in Aerospace is due to many reasons. While the properties of metallic materials are mastered, the risk behavior of composite materials requires monitoring of the structure. That's why many methods of non-destructive testing (NDT) have been implemented and applied for the evaluation of defects in composites.

The purpose of our research is the development of a new NDT tool for detecting delamination damages in aircraft's structures. The principle diagnostic is based on the application of a reproducible impact wave on a composite panel and its propagation trough an accelerometer sensor network. The presence of defects will be identified by the temporal and frequency response change compared to calibrated reference recorded before the damage.

Therefore, in this paper an experimental study of impact behavior of a composite laminate structure will be presented. The panel is made from a guarded composite long carbon fiber laminate. In the study, the shock propagation wave induced in the materials will take into account the fiber orientation $\left(0^{\circ}, 45^{\circ}, 90^{\circ},-45^{\circ}\right)$ and plies arrangement (plies stacking sequence of tilt-up). First results of this new NDT for detecting damages of an aircraft structure composite demonstrate the wide possibilities of this technique.
\end{abstract}

Key words: Structural Health Monitoring, Impact detection, Non Destructive Testing, Composite laminate, Accelerometer detection.

\section{INTRODUCTION}

Small defects, as delamination, micro-cracks or fiber damage in aeronautic composite materials, can have severe consequences on the reliability of a mechanical structure, of blade plane or more generally on the aircraft. Contrary to the defects on the surface, which are easily identifiable by simple observation, these anomalies are invisible during monitoring visits because they are in the volume of the structure.

However, when these structures assume real working and environmental conditions (stresses and vibrations), these defects can lead to structural failure (break down). It is therefore essential to detect the presence of any abnormality. It can be done using NDT methods. The NDT is a set of methods used in science and industry to evaluate (characterize) the properties of a material, component or system without degrading them, either during production, in use and in the stage of maintenance. The terms NonDestructive Examination (NDE) [1], NonDestructive Inspection (NDI) [2], and NonDestructive Evaluation (NDE) are also commonly used to describe this approach. 
Because NDI does not undergo a change on the structure being inspected, it is a potential and reliable method that can save both money and time in product's reliability evaluation. There are many techniques of NDT. However, we can differentiate four main methods: The ultrasounds [3]; the currents of Foucault; the tomography with X-rays [4] and the Lamb wave $[5,6]$. These methods are commonly used in medical (sonogram), aeronautic (structure monitoring), art (check the sticking), automotive (quality of the welds)...etc.

Many multilayer composite structures are exceptionally difficult to examine using standard or conventional NDT methods, such as the generally employed ultrasonic methods, due to their large surface, complicate accessibility. Shock methods provide a potential solution.

The objective of this work is to develop a new NDT tool for detecting delamination damages in aircraft composite structures (carbon/epoxy HTA-E25 from an Airbus ATR72 aircraft); by deploying on the skin of the structure a network of accelerometers and by comparing their responses to a reference shock [7].

\section{OUR APPROACH}

Our objective is to detect damages within a composite material by vibration technique [8]. It consists of throwing a calibrate impact energy on the panel and read its wave propagation through the accelerometers' network.

The presence of defects will be identified by comparing the temporal and frequency recorded responses of the sensors before (reference) and after damage. The flowchart shown in Fig.1 illustrates our methodology for defects' detection. This includes:

Impact calibration: find the reference energy corresponding to the lowest impact which allows us to have a response without damage (reading wave).

$>$ Record the accelerometers' responses before creation of the defects.

Creation of defects with different sizes.

$>$ Reading of accelerometers' responses after creation of defects.

$>$ Post processing: treatment and comparison of signals recorded before and after damages.

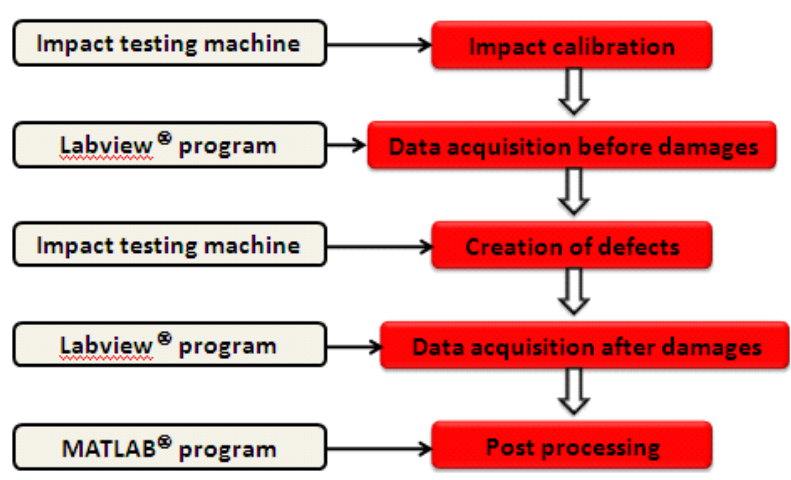

Fig. 1. The current approach for defect detection 


\section{EXPERIMENTAL}

\section{Device presentation}

To support our work we have applied our approach on a composite mechanical structure. It consists of a panel extracts from the intrados of the right wing of an ATR72. The panel is made of carbon/epoxy HTA-EH25 laminate composite, including four ribs and measures 460 x $498 \mathrm{~mm}^{2}$. Figure and table 2 illustrate respectively, the bottom view of the panel and the properties of the composite material.

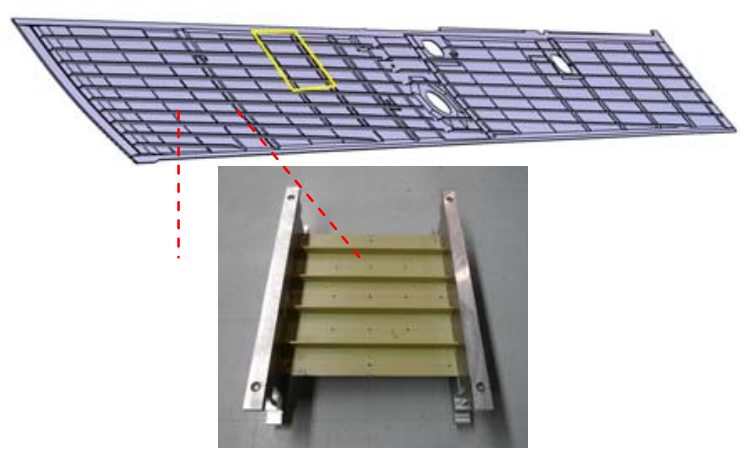

Table 1. Composite material properties

\begin{tabular}{ll}
\hline properties & Values \\
\hline & \\
$\mathrm{E}_{1}[\mathrm{GPa}]$ & 138 \\
$\mathrm{E}_{2}[\mathrm{GPa}]$ & 9.8 \\
$v_{12}[-]$ & 0.28 \\
$\rho\left[\mathrm{g} / \mathrm{cm}^{3}\right]$ & 1.586 \\
$\mathrm{G}_{12}, \mathrm{G}_{13}, \mathrm{G}_{23}[\mathrm{GPa}]$ & 5.03 \\
Folds numbers & 22 \\
Thickness of folds $(\mathrm{mm})$ & 0.125 \\
& \\
\hline
\end{tabular}

\section{Test bench description}

The damage detection has been realized using the test bench presented in figure 3 . This setup measures the evolution of the propagation's wave over time for a given impact (shock). It is composed by two essential parts having each one a particular function: The first one, concerns the actuation part. It consists of a programmable impacting machine, used to apply different shocks through a hemispherical hummer with a diameter of $16 \mathrm{~mm}$ and weight of $118 \mathrm{~g}$. 


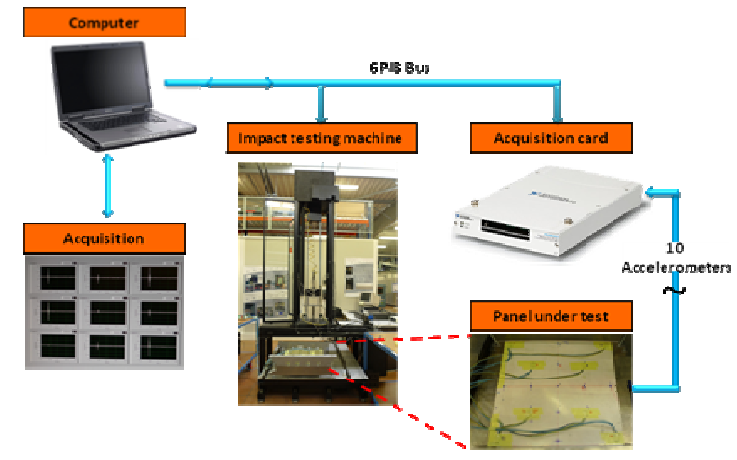

Fig. 3. Non destructive test bench

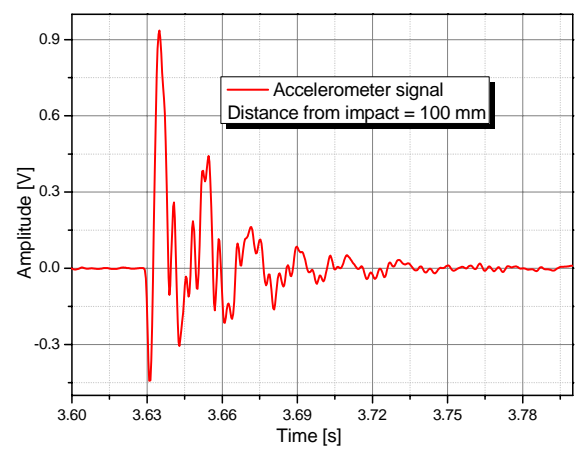

Fig. 4. Accelerometer response

The second part, allows measuring the evolution of the propagation's wave in time by means of sensor network (MMA6281QT 2.5g-10g, sensitivity $120-480 \mathrm{mV} / \mathrm{g}$ ). These accelerometers are glued on the internal or external surface of the panel according to the realized experience. To ensure the good progress of the tests, the structure is maintained at both extremities.

A LabView program was written to automate the test bench: it measures 10s after throwing a shock, eight parallel accelerometers (Fig. 4).

\section{EXPERIMENTAL RESULTS}

To understand the wave impact propagation over the composite panel, we placed the accelerometers in the configuration of figure 5. By making an impact at the center of the panel, we can assume:

$>$ the wave impact propagates symmetrically relative to the axis $0^{\circ}$ of draping,

$>$ the rib provokes attenuation of the shock wave (results given by the radar curves Fig. 6).

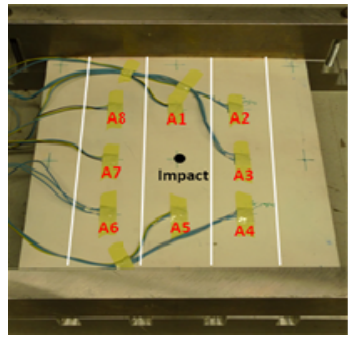

(a)

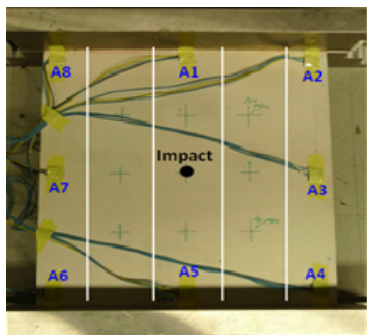

(b)
Fig. 5. (a) Configuration 1, (b) Configuration 2

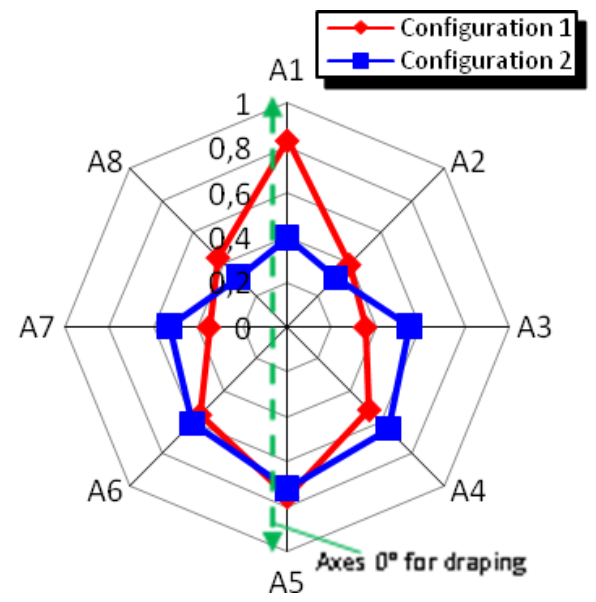

Fig. 6. Shock wave propagation of the signal amplitude 
Before performing the tests on the mechanical structure, it is necessary to determine the impact reference. This last one corresponds to the lowest impact which allows us to have a response without any damage. Once this reference calibrate, we set our accelerometer network on the external face of the panel as shows in figure 7 . The sensors are spaced out by an interval of $145 \mathrm{~mm}$. The first test consists in realizing one impact in the center of the panel with reference energy ( 3 Joules) and to record the responses of the eight accelerometers. These registered data will serve as reference (before damage).

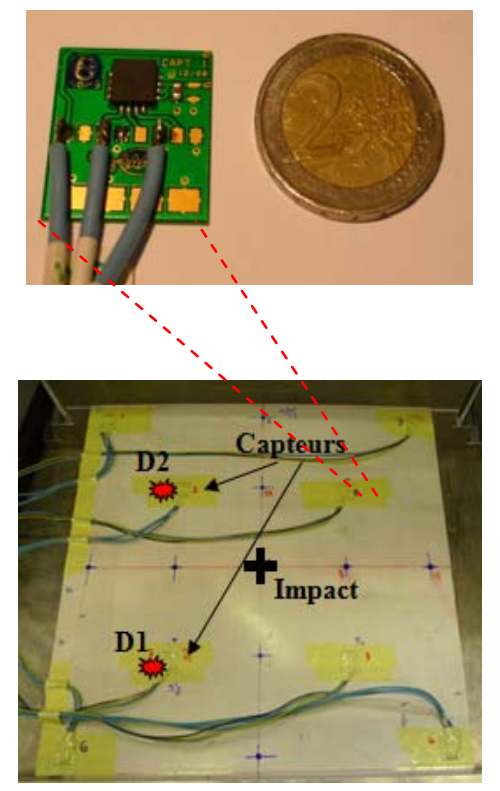

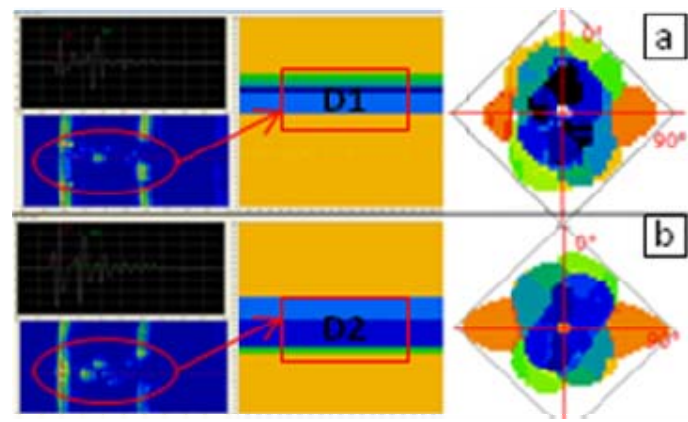

Fig. 8. Ultrasonic C-Scan mapping

Fig. 7. Panel under test

The validation of our approach requires the creation of defects on the mechanical structure. To do this we increased intentionally the impact energy to 5 and 10 joules respectively on two points, respectively D1 and D2 as shown in figure 7. By drawing an ultrasound map (hard to implement), we have verified the creation of these defects (Fig.8). During this check we observed a defect on D1 (10 joules), however in D2 the damage was been created after a three series of 5 joules tests. The characteristics of these damages are depicted in table 2 .

Table 2. Tests conditions

\begin{tabular}{cccc}
\hline & Ref & D2 & D1 \\
\hline & & & \\
Impact energy [J] & 3 & $5+5+5$ & 10 \\
Fall height [mm] & 91 & 154 & 303 \\
Impact speed [m/s] & 1.35 & 1.9 & 2.45 \\
Total mass [Kg] & 3.4 & 3.4 & 3.4 \\
Clamping [Nm] & 12 & 12 & 12 \\
Damage surface [mm $\left.{ }^{2}\right]$ & - & 188.5 & 320
\end{tabular}


The next task in our approach is to determine the defects' influences (signatures) on the signal wave propagation. Therefore, we tried to observe the variations of certain parameters.

Three parameters have been selected for this study: the maximum amplitude Amax, the Mean $\mathrm{M}$ and the mean square value RMS of the signal.

The results obtained during the tests, allow us to observe that the creation of a defect in the composite structure does not attenuate the signal responses of the accelerometers, but it increases the order of the frequency response, as shown in observed oscillations (Fig. 9).
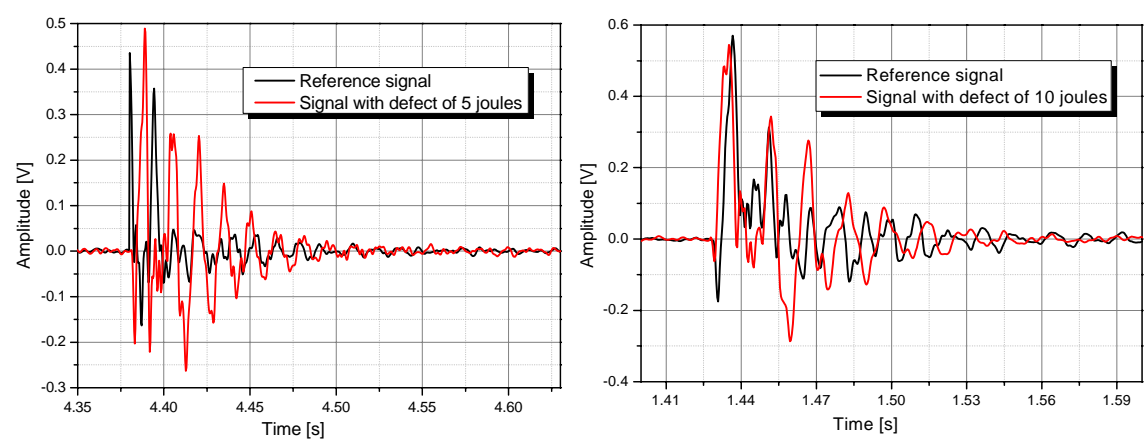

Fig. 9. Accelerometers response with and without defect (fs $=25 \mathrm{kHz}$ )
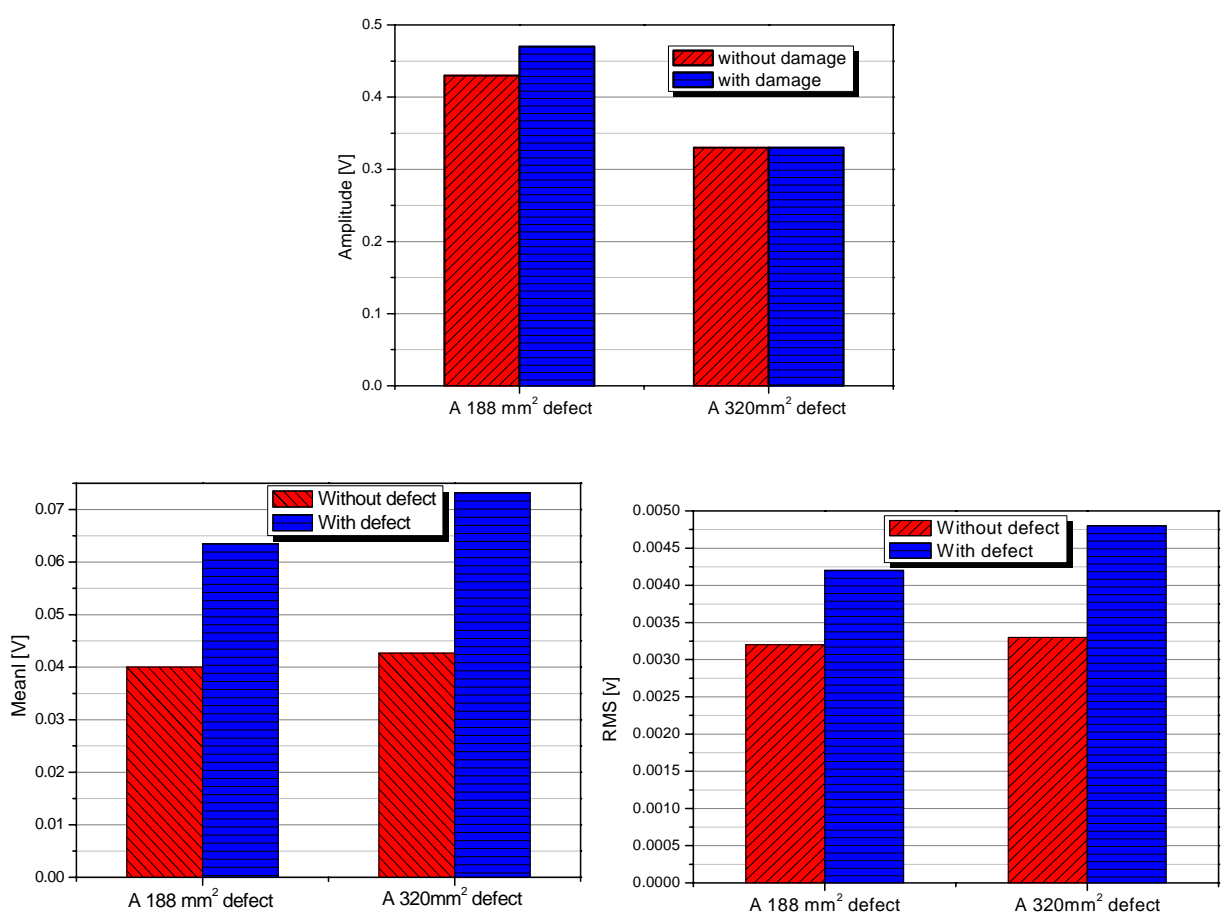

Fig. 10. Comparative histogram of the signals parameters

Through a quantitative analysis of these results, we can deduce that the maximum amplitude is not a criterion to be held (retained) because it remains constant (before and after defects). However the average of the Mean and the RMS signal increase in the presence of damages as shown in table 3 and figure 10. 
Table. 3. Rate of signal parameters change

\begin{tabular}{ccccc} 
Defects & Dimension & $\boldsymbol{A}_{\max }$ & $\boldsymbol{R}$ & $\boldsymbol{M}$ \\
\hline $\mathrm{D} 1$ & $188 \mathrm{~mm}^{2}$ & - & $\boldsymbol{\lambda} 34 \%$ & $\boldsymbol{\lambda} 33 \%$ \\
\hline $\mathrm{D} 2$ & $320 \mathrm{~mm}^{2}$ & - & $\boldsymbol{\lambda} 27 \%$ & $\boldsymbol{\lambda} 33 \%$ \\
\hline
\end{tabular}

\section{CONCLUSION}

We demonstrate in this paper the possibility offered by the proposed approach to identify damage in laminate composite structure. This method is based on a comparative analysis of the accelerometers responses to the calibrated impact (here 3 Joules). In future works we focus on the localization of the defect and on the influence of the surface damage on the signal parameters.

\section{ACKNOWLEDGEMENTS}

This work was supported by DIAGNOSTAT project, in collaboration with EADS-IW, Airbus and other research laboratories. It was made possible by funding from French "Ministère Economie et des Finances" and "Région Midi Pyrénées".

\section{REFERENCES}

1. J. Higgins, B. VanWest, NDE and repair of damaged Minotaur fairing shell, Composite Structures, Vol 67, 189-195, 2005.

2. R. Ruzek, R. Lohonka, J. Jironc, Ultrasonic C-Scan and shearography NDI techniques evaluation of impact defects identification, NDT\&E International, Vol 39, 132-142, 2006.

3. M. V. Hosur, C. R. L. Murthy, T. S. Ramamurthy, Anita Sheb, Estimation of impact-induced damage in CFRP laminates through ultrasonic imaging. NDT\&E International, Vol.31, No.5, 359-374, 1998.

4. Y.Y. Hung, Y.S. Chen, S.P. Ng, L. Liu, Y.H. Huang, B.L. Luk, R.W.L. Ip, , C.M.L. Wu and P.S. Chung, Review and comparison of shearography and active thermography for nondestructive evaluation, Materials Science and Engineering, Vol. 64, No. 5-6, 73-1121, 2009.

5. R. Kažys, R.Raišutis, E. Žukauskas, L. Mažeika, A.Vladišauskas, Air- coupled ultrasonic testing of CFRP rods by means of guided waves, Physics Procedia, Vol. 3, 185-192, 2010.

6. S. Yashiro, J. Takatsubo, N. Toyama, An NDT technique for composite structures using visualized Lamb-wave propagation, Composites Science and Technology 67, 3202-3208, 2007.

7. H. Boukabache, S. Ksouri, C. Escriba, J.Y. Fourniols, Piezoelectric sensor/actuator network for SHM and damage detection on Airplane Door, Asia-Pacific Workshop on Structural Health Monitoring, Tokyo, Japan, 2010

8. C. Escriba, H. Boukabache, S. Ksouri, , M. Lastapis, T. Camps, J.M. Dilhac, S.Rolet, J.Y. Fourniols, Sensor Network for non destructive control of aeronautics structures: piezo or accelerometer diagnosis?, European Workshop on Structural Health Monitoring, Sorrento, Italy, 2010, pp. 388-393. 